\title{
Infrared Spectroscopy of GaAs Doped with Mn
}

\author{
A. Rutkowska ${ }^{a}$, D. $\operatorname{Wasik}^{a}$, A. Witowski ${ }^{a}$, M. $\operatorname{Sadowski}^{b}$, \\ W. Orlowski ${ }^{c}$, G. StrzeleckA ${ }^{c}, A \cdot$ Hruban $^{c}$, M. Kamińska ${ }^{a}$, \\ A. Twardowski ${ }^{a}$ AND M. Potemski ${ }^{b}$ \\ ${ }^{a}$ Institute of Experimental Physics, Warsaw University \\ Hoża 69, 00-681 Warsaw, Poland \\ ${ }^{b}$ Grenoble High Magnetic Field Laboratory \\ 38042 Grenoble Cedex 09, France \\ ${ }^{c}$ Institute of Electronic Materials Technology \\ Wólczyńska 133, 01-919 Warsaw, Poland
}

\begin{abstract}
We found that the fine structure related to Lyman spectra of $\left[\mathrm{Mn}^{2+}\left(d^{5}\right)\right.$ + a hole] centers in GaAs was present only for samples with low Mn concentration. Such samples, at low temperature, did not show any hopping conductance within Mn impurity band. Magnetooptical measurements revealed that magnetic field induced splitting of the Lyman optical transitions was larger than Zeeman splitting observed for typical shallow acceptors in GaAs, like Be, Zn, and C. This experimental result proved that in the case of Mn acceptor impurity, the exchange coupling of a hole and the $S=5 / 2$ $\mathrm{Mn}^{2+}\left(d^{5}\right)$ core could not be neglected, which was in accordance with the $\left[\mathrm{Mn}^{2+}\left(d^{5}\right)+\right.$ a hole $]$ model of the neutral Mn center in GaAs.
\end{abstract}

PACS numbers: 71.70.Ej, 78.20.Ls, 78.30.Fs

\section{Introduction}

Recently a lot of attention has been devoted to III-V semiconductor compounds doped with $\mathrm{Mn}$, since according to theoretical prediction they could be ferromagnetic at room temperature [1]. If $\mathrm{Mn}$ ion substitutes for $\mathrm{Ga}$ in $\mathrm{GaAs}$ lattice it has to give three electrons to the bonds. Therefore, the most natural seems to be $\mathrm{Mn}^{3+}$, neutral configuration of manganese with four electrons bound on $d$ shell $\left(d^{4}\right)$. However, optical and electron paramagnetic resonance experiments have shown that energetically more favorable is a configuration of $\mathrm{Mn}^{2+}$ 
with half-filled $d$ shell $\left(d^{5}\right)$ and such locally negatively charged Mn ion acts as an acceptor which coulombically bounds a hole $\left[\mathrm{Mn}^{2+}\left(d^{5}\right)+\right.$ a hole center $][2,3]$. If the concentration of Mn ions is low enough, intra-acceptor optical transitions between ground and excited states of the hole should be seen as discrete lines on continuous background originating from photoionization transition of the center.

Indeed, such spectrum has been reported in [4] and the effects of magnetic field and uniaxial stress on one of the discrete lines have been studied there. The ionization energy of $\left[\mathrm{Mn}^{2+}\left(d^{5}\right)+\right.$ a hole $]$ center has been determined as about $113 \mathrm{meV}$ [5], much higher than for typical shallow acceptors in GaAs (about $20 \mathrm{meV}$, see Table I and II). Therefore, the questions arise if such large chem-

TABLE I

Transition energies from the $1 S_{3 / 2}\left(\Gamma_{8}\right)$ ground state to $n p$ excited states (in $\mathrm{meV}$ ).

\begin{tabular}{c|c|c|c|c}
\hline \hline Final state & $\mathrm{Mn}$ & $\mathrm{C}[4]$ & $\mathrm{Zn}[4]$ & $\mathrm{Be}[3]$ \\
\hline $2 P_{3 / 2}\left(\Gamma_{8}\right)$ & 101.08 & 15.19 & - & 16.66 \\
$2 P_{5 / 2}\left(\Gamma_{8}\right)$ & 105.16 & 19.35 & 23.13 & 20.68 \\
$2 P_{5 / 2}\left(\Gamma_{7}\right)$ & 107.25 & 21.19 & 24.92 & 22.60
\end{tabular}

TABLE II

Energy separation (in meV) between $n p$ excited states determined from the Lyman transition (see Table I).

\begin{tabular}{l|c|c|c|c}
\hline \hline & $\mathrm{Mn}$ & $\mathrm{C}[4]$ & $\mathrm{Zn}[4]$ & $\mathrm{Be}[3]$ \\
\hline $2 P_{5 / 2}\left(\Gamma_{8}\right)-2 P_{3 / 2}\left(\Gamma_{8}\right)$ & 4.08 & 4.16 & - & 4.02 \\
$2 P_{5 / 2}\left(\Gamma_{7}\right)-2 P_{5 / 2}\left(\Gamma_{8}\right)$ & 2.09 & 1.84 & 1.79 & 1.92
\end{tabular}

ical shift also affects splitting of all the excited states in magnetic field, and how the exchange interaction between $d^{5}$ localized spin and spin of weakly bound hole influences the splitting of the levels. questions.

The aim of the presented paper was to try to experimentally answer these

\section{Experimental details and results}

GaAs crystals, intentionally doped with Mn, were grown by the Czochralski method. Manganese was introduced to the melt. The samples were characterized by electron transport measurements. The free hole concentration at room temperature was used as a measure of Mn content in the sample [6].

Optical absorption measurements were performed at about $10 \mathrm{~K}$, using a Fourier transform spectrometer Bruker IFS 113V. Crystallographically oriented samples were prepared for the magnetotransmission experiment. They were cut from the same ingot, with plane perpendicular to different crystallographic direc- 
tions: (100), (110), (111), and (112). The samples were measured in magnetic field up to $11 \mathrm{~T}$ at about $4.5 \mathrm{~K}$, in Faraday geometry (magnetic field $\boldsymbol{B}$ parallel to electromagnetic wave vector and normal to the sample plane). The unpolarized light was applied.

Optical absorption experiments showed that the intra acceptor transitions of $\left[\mathrm{Mn}^{2+}\left(d^{5}\right)+\right.$ a hole] center were clearly observable exclusively for samples with low Mn composition, of the order of $10^{16} \mathrm{~cm}^{3}$. For higher Mn content, the spectra revealed only the photoionization absorption.

This is shown in Fig. 1, in which the absorbance spectra of two samples with a slightly different $\mathrm{Mn}$ content in the spectral region of intra-acceptor transition are presented. Only one of the samples shows a fine structure related to Lyman transitions of $\left[\mathrm{Mn}^{2+}\left(d^{5}\right)+\right.$ a hole $]$ centers.

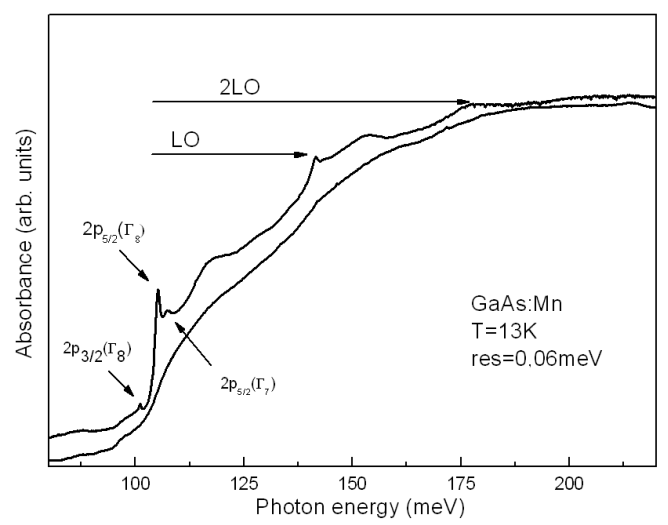

Fig. 1. Infrared absorption spectra of two GaAs:Mn samples having comparable free hole concentration (about $3 \times 10^{16} \mathrm{~cm}^{-3}$ at room temperature).

The three lines due to the transitions between ground $1 S_{3 / 2}$ and excited $2 P$ states with different total momentum $J$, originating from different valence subbands (namely $\Gamma_{8}$ and $\Gamma_{7}$ ) are clearly seen. It is found that relative intensities of the lines are in agreement with those of the corresponding lines observed for shallow acceptors of GaAs $[7,8]$.

The magnetotransmission results, shown in Fig. 2 for (110) sample orientation, are almost the same for all the measured sample orientations. The splitting of weak lines is clearly visible. The absorption maxima as a function of magnetic field are plotted in Fig. 3. As can be seen in Figs. 2 and 3, the line corresponding to the $1 S_{3 / 2}\left(\Gamma_{8}\right) \rightarrow 2 P_{3 / 2}\left(\Gamma_{8}\right)$ optical transition splits into three components and the splitting is linear with magnetic field. The strongest line, $1 S_{3 / 2}\left(\Gamma_{8}\right) \rightarrow 2 P_{5 / 2}\left(\Gamma_{8}\right)$ transition, splits into five components, which is in agreement with Ref. [4]. Its highest energy component clearly shows a nonlinear dependence on magnetic field. The last line, corresponding to $1 S_{3 / 2}\left(\Gamma_{8}\right) \rightarrow 2 P_{3 / 2}\left(\Gamma_{7}\right)$ transition, reveals a more complicated behavior. Its low energy components merge with $2 P_{5 / 2}\left(\Gamma_{8}\right)$ lines. 


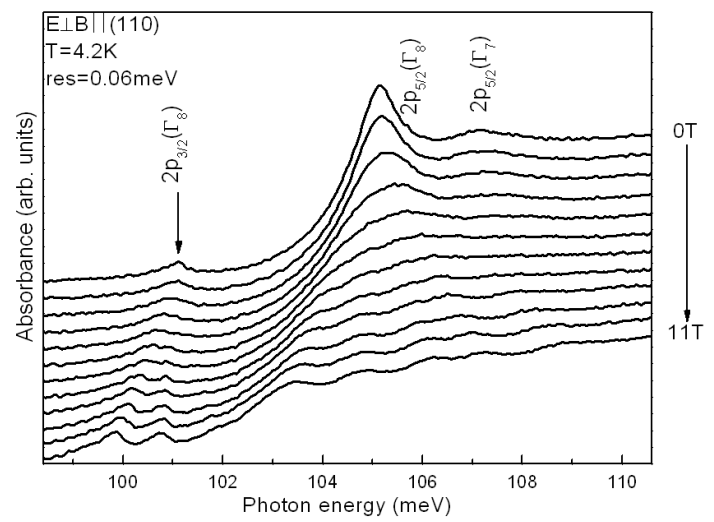

Fig. 2. Evolution with magnetic field of the Lyman spectra for a chosen crystallographic orientation.

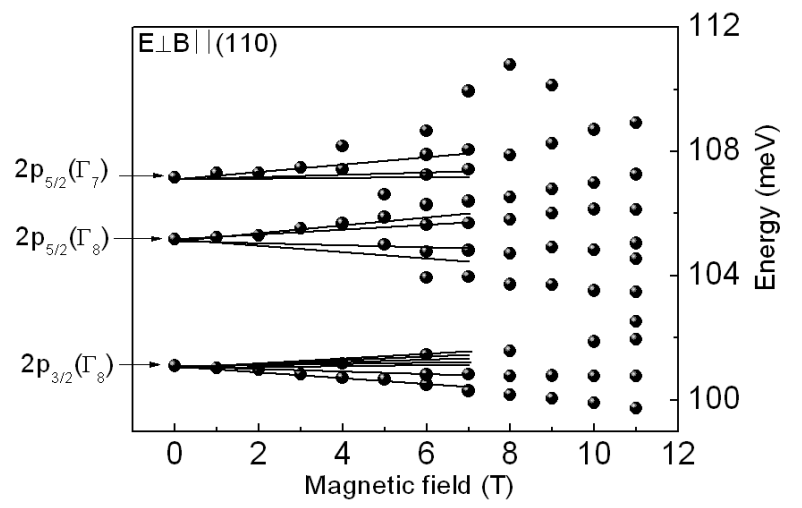

Fig. 3. Zeeman splitting of the Lyman transitions for magnetic field parallel to (110) crystallographic orientation. The rescaled data for carbon taken after Ref. [7] are presented as straight lines.

Therefore, in some ranges of magnetic field, they show a nonlinear magnetic field dependence.

\section{Discussion}

We found that the fine structure related to Lyman transitions of $\left[\mathrm{Mn}^{2+}\left(d^{5}\right)\right.$ + a hole] centers was observed only for samples which, at low temperature, did not show any hopping conductance within Mn impurity band. This corresponded to a low enough concentration of Mn centers where no overlapping of their wave functions occurred [6].

For the line $1 S_{3 / 2}\left(\Gamma_{8}\right) \rightarrow 2 P_{5 / 2}\left(\Gamma_{8}\right)$ the experimental results presented in this paper are compatible with those reported in Ref. [4], but measured only for magnetic fields up to $6 \mathrm{~T}$. To estimate the effect of possible exchange interaction of the acceptor bound hole with electrons from Mn $\left(3 d^{5}\right)$ shell, in Fig. 3 we plotted 
rescaled data for a typical shallow acceptor in GaAs, like carbon after Ref. [7] together with the results for $\left[\mathrm{Mn}^{2+}\left(d^{5}\right)+\right.$ a hole] center. As it is seen, the above-mentioned interaction amplifies the effect of the magnetic field. The observed splitting for $\left[\mathrm{Mn}^{2+}\left(d^{5}\right)+\right.$ a hole] center is at least $50 \%$ larger than for a typical shallow acceptor in GaAs. Therefore, the interaction between bound hole and the electrons of $d^{5}$ shell cannot be neglected in theoretical description of Mn acceptor.

\section{Summary}

Our results agree with the model of $\left[\mathrm{Mn}^{2+}\left(d^{5}\right)+\right.$ a hole $]$ center, where an additional electron is incorporated into the Mn impurity to the half-filled $d$ shell ( $d^{5}$ configuration) and such negatively charged center binds a free hole on the acceptor state.

\section{Acknowledgments}

This work was partially supported by PBZ-KBN-044/P03 and G5RD-CT2001-00535 grants.

\section{References}

[1] T. Dietl, H. Ohno, F. Matsukura, J. Cibert, D. Ferrand, Science 287, 1019 (2000).

[2] R.A. Chapman, W.G. Hutchinson, Phys. Rev. Lett. 18, 443 (1967).

[3] J. Schneider, U. Kufmann, W. Wilkening, M. Baeumler, Phys. Rev. Lett. 59, 240 (1987).

[4] M. Linarsson, E. Jenzén, B. Monemar, M. Kleverman, A. Thilderkvist, Phys. Rev. B 55, 6938 (1997).

[5] Enver Tarhan, I. Miotkowski, S. Rodriguez, A.K. Ramdas, Phys. Rev. B 67, 195202 (2003).

[6] M. Pawłowski, M. Piersa, A. Wołoś, M. Palczewska, A. Strzelecka, A. Hruban, J. Gosk, M. Kamińska, A. Twardowski, Acta Phys. Pol. A 108, 825 (2005).

[7] R.A. Lewis, Y.-J. Wang, M. Henini, Phys. Rev. B 67, 235204 (2003).

[8] R. Atzmüller, M. Dahl, J. Kraus, G. Schaack, J. Schubert, J. Phys., Condens. Matter 3, 6775 (1991). 